\title{
Thermal stability of organic carbon in soil aggregates of maize- wheat system in semi arid India
}

\author{
S. Sandeep* and K.M. Manjaiah \\ Division of Soil Science and Agricultural Chemistry, Indian Agricultural Research Institute, New Delhi -110 012, India. \\ ${ }^{*}$ Corresponding author: sandeepagri@gmail.com
}

\begin{abstract}
Understanding the stability and carbon retention ability of aggregates under common cropping sequences is essential to ascertain terrestrial carbon storage. Surface soil samples $(0-15 \mathrm{~cm})$ were collected and dry sieved to separate the macro $(>250 \mu \mathrm{m})$ and micro sized aggregates $(<250 \mu \mathrm{m})$. The separated aggregates were subjected to 60 days incubation at four different temperatures $\left(25,30,35\right.$ and $\left.40{ }^{\circ} \mathrm{C}\right)$. Nutrient management was more prominent than tillage in determining water stable aggregates. $100 \%$ organic treated plots in bed planting and mineral fertilizers + crop residues in conventional tillage gave maximum mean weight diameter values of 1.01 and 0.95 respectively. Irrespective of the treatments, microaggregates were found to be poorer in carbon content, but richer in their capacity to retain it by way of larger activation energies. The $\mathrm{Q}_{10}$ values were found to be higher in microaggregates than macroaggregates for all treatments, confirming the thermal sensitivity hypothesis of stable carbon fractions.
\end{abstract}

Keywords: Tillage, nutrient management, macro and microaggregates, aggregate stability, thermal stability

\section{Introduction}

Soils in the semi arid tropics are (generally) low in organic matter and nutrient reserves. High temperature in these regions causes rapid decomposition and loss of organic matter and is one of the major causes for its low status in these soils (Garten, 2011). Besides, under dry land agriculture, application of nutrients and organic matter through external inputs is low due to socioeconomic conditions of the farmers, lower institutional credit support and subsistence farming practices. Despite low external additions and fast carbon decomposition, these regions assume particular interest with respect to percentage of global soil organic carbon (SOC) stocks. So processes and factors of carbon dynamics of these soils have been a subject of intense investigation and such studies carry with it ramifications on atmosphere - biosphere $\mathrm{CO}_{2}$ exchange and influence of this reciprocal flux for global climate change (Aggarwal et al. 2006; Houghton, 2007; Bhattacharya et al. 2010).

Soil aggregate formation is a major mechanism for carbon storage and enrichment in soils (Eynard et al. 2006). At a microscopic scale, sand and silt particles are bridged by clay and organic matter to produce microaggregates (McLaren and Cameron, 1996). The soil microaggregates are bound together into macroaggregates by fungal hyphae, plant roots and other stabilizing agents (Six et al. 2004). 
Carbon within aggregates is protected physically, chemically or biologically and has longer turnover time than unprotected forms. In other words, the SOC distribution in soil aggregates controls the sequestration or release of $\mathrm{C}$ within a given agricultural system (Han et al. 2010). Carbon dynamics in the aggregate is influenced by its recalcitrance and accessibility, and interactions between organic carbon (OC) and soil components (Swanston et al. 2002). Land use and land use changes affect aggregation in a way that the proportion of water stable macroaggregates are reduced under the practices with extensive soil disturbance (Ashagrie et al. 2007). Besides, tillage intensity, addition of manures and fertilizers, crop rotation, and climate are the important factors that contribute to the buildup or loss of SOC under arable agriculture. Microaggregates, however, seem to be less influenced by such changes and hence offer more protection to the stored carbon (Zheng et al. 2011).

Among the different climatic parameters, temperature is a major rate deciding factor of organic carbon decomposition in soils (Kirschbaum, 1995). As global temperatures rise, changes in soil $\mathrm{CO}_{2}$ emissions will in part be determined by the temperature dependence of soil carbon decomposition. The short-term temperature dependence of decomposition in soils is well established experimentally (Knorr et al., 2005), and is modelled either by a constant $\mathrm{Q}_{10}$ value of $\sim 2$ or more accurately by the Arrhenius equation. The Arrhenius function shows an increase in the temperature sensitivity of decomposition with stability of organic compounds as stabilised substrates are less reactive due to their higher activation energies (Davidson and Janssens, 2006). A perusal of the above observations leads to the conclusion that soil organic carbon held by aggregates (e.g. recalcitrant or adsorbed or complexed SOC) are characterised by low decomposition rates, high activation energies and, therefore, 'inherently' high temperature sensitivity. But contradictions exist to the above concepts and it has been suggested that soil carbon pools may be depleted much less than that is predicted by most ecosystem models (Giardina and Ryan, 2000). In the absence of a consensus on the temperature sensitivity of decomposition of a large fraction of soil C stocks, the significance of this positive feedback continues to be debated. SOC stocks in the semi arid regions are highly vulnerable to decomposition due to prevailing environmental conditions. Assessment of carbon stocks in the semi arid tropics often neglects the temperature sensitivity analysis of stored carbon and hence lacks valuable insight into soil carbon dynamics of these regions.

A field experiment was initiated in 2001 at Indian Agricultural Research Institute's farm, New Delhi to study the influence of tillage and nutrient management practices on soil health and crop productivity under maize-wheat cropping sequence, a common cropping sequence of Indo - Gangetic Plains of India. Nutrient management treatments involving organic sources were found to maintain the SOC levels without further deterioration, but couldn't improve these significantly above their initial levels (data not shown). Hence we made an attempt to outline the thermal stability of SOC under different management practices in this maize - wheat rotation and thereby evolve a better treatment combination for carbon storage in these soils. The objectives of the present study can be stated as (1) to determine the effects of tillage and nutrient management on soil aggregation in a maize - wheat system, and (2) to study the thermal stability of carbon in the macro $(>250 \mu \mathrm{m})$ and micro $(<250 \mu \mathrm{m})$ sized soil aggregates of this cropping system.

\section{Materials and Methods}

\subsection{Location, soil sampling and processing}

The study area falls under semi-arid and subtropical zone characterized by hot summer and cold winter. Mean annual precipitation is about $650 \mathrm{~mm}$, most of which is received during the monsoon period of July to September. The annual maximum temperature is $35^{\circ} \mathrm{C}$, while the mean minimum temperature is $18^{\circ} \mathrm{C}$. 
Experimental soil is loam in texture and belongs to Holambi series, Typic Haplustepts (Inceptisols, USDA). Initial $\mathrm{pH}$, clay, and organic carbon content were $7.7,25 \%$, and $0.40 \%$, respectively. The experiment was laid out in split plot design with three replications. Two tillage treatments and six manurial /fertilizer treatments were allocated to main and subplots, respectively (Table 1). All the treatments including control have been receiving recommended dose of $\mathrm{P}\left(26.2 \mathrm{~kg} \mathrm{ha}^{-1}\right)$ and $\mathrm{K}(50$ $\mathrm{kg} \mathrm{ha}^{-1}$ ) through single super phosphate (SSP) and muriate of potash (MOP), respectively, except $T_{6}$. Farmyard manure (FYM) was incorporated 15 days before sowing of crops. After separating the grain, crop residues were retained in the field under specific plots, which constitutes the treatment $\left(\mathrm{T}_{5}\right)$ for the succeeding crops. For biofertilization, Azotobacter sp. W5 strain was applied on the seed at the time of sowing of maize as a carrier-based culture at the rate of $10^{9}$ cells $/ \mathrm{g}$. Similarly, Azospirilum sp. CD strain was used for wheat.

Soil samples from surface $(0-15 \mathrm{~cm}$ depth) were collected during October/November 2006 (after the harvest of Maize). Soil samples were air-dried, ground, and passed through 2-mm sieve. $500 \mathrm{~g}$ of this processed sample was passed through a $250 \mu \mathrm{m}$ sieve to separate macro $(>250 \mu \mathrm{m})$ and microaggregates $(<250 \mu \mathrm{m})$ and used for studying the carbon stabilization in them (Mendes et al. 1999). The organic carbon content in the soils and aggregates was estimated by wet-oxidation method (Walkley and Black, 1934).

The stability of the soil aggregates expressed as mean weight diameter (MWD) was deduced by wet sieving of soils. There were five size classes used: $<0.25$, $0.25-0.5,0.5-1.0,1.0-2.0$ and $2.0-4.0 \mathrm{~mm}$. The mean weight diameter (MWD) of the soil aggregates was calculated according to the following formula:

$$
\mathrm{MWD}=\sum_{j=1}^{k} W j \overline{\mathrm{X}}
$$

where, MWD = mean-weight diameter, $(\mathrm{mm}) ; \overline{\overline{x_{1}}}=$ arithmetic mean diameter of the $\mathrm{j}-1$ and $\mathrm{j}$ sieve openings $(\mathrm{mm}) ; W j=$ proportion of the total sample weight (uncorrected for sand and gravel) occurring in the fraction (dimensionless); and $\mathrm{k}=$ total number of size fractions (in this case 5).

\subsection{Thermal stability of carbon in macro $(>250 \mu \mathrm{m})$ and micro $(<250 \mu \mathrm{m})$ sized soil aggregates}

Temperature responses of carbon in aggregates (macro and micro) were investigated for 60 days. $25 \mathrm{~g}$ of aggregates were incubated in wide mouth bottles after estimating the initial OC (\%) and moistening it to field capacity which was maintained throughout the experiment. The mouths of the bottles were kept open during the experiment period to enable gaseous exchange. Four incubation temperatures viz. 25, 30, 35 and $40^{\circ} \mathrm{C}$ were used for the study. Six sets of samples and three replications were used for each temperature and at the end of incubation period $\left(5^{\text {th }}, 10^{\text {th }}, 20^{\text {th }}, 30^{\text {th }}\right.$ $45^{\text {th }}$ and $60^{\text {th }}$ day) each set was removed and organic carbon remaining in samples estimated by Walkley and Black method (Organic carbon (OC) referred to in this study is WBC). OC lost during the period was calculated by subtracting remaining amount from initial organic carbon content of aggregates (at $0^{\text {th }}$ day) and was used for determining first order rate kinetics using the equation:

$$
\mathrm{A}_{\mathrm{t}}=\mathrm{A}_{0} \mathrm{e}^{-\mathrm{kt}} \quad \text { Eq. (1) }
$$

where, $\mathrm{k}$ is the reaction rate constant; $\mathrm{A}_{0}$ and $\mathrm{A}_{\mathrm{t}}$ are the amount of organic carbon at zero and ' $\mathrm{t}$ ' time. Activation energy was worked out using Arrhenius equation:

$$
\mathrm{k}=\mathrm{A} \exp (-\mathrm{Ea} / \mathrm{RT}) \quad \text { Eq. (2) }
$$

where, $\mathrm{k}$ is the reaction rate constant; $\mathrm{A}$ is the frequency factor; $\mathrm{Ea}$ is the required activation energy in joules per mole; $\mathrm{R}=8.314 \mathrm{~J} \mathrm{~K}^{-1} \mathrm{~mol}^{-1}$ gas constant and $\mathrm{T}$ is the temperature. Activation energy was worked out from the slope $(\mathrm{Ea} / \mathrm{R})$ obtained by plotting $\ln \mathrm{k}$ against 1/T (Knorr et al. 2005). 
$\mathrm{Q}_{10}$ indicates response of biological processes with temperature. $\mathrm{Q}_{10}$ was calculated as a function:

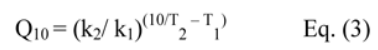

where $\mathrm{k}_{2}$ and $\mathrm{k}_{1}$ are the reaction rates at temperatures $\mathrm{T}_{2}$ and $\mathrm{T}_{1}$ respectively (Kirschbaum, 1995).

\subsection{Statistical analysis}

Analysis of variance (ANOVA) for split - plot design was performed using SAS (version 9.3) software with tillage as the main plot treatment and nutrient management as subplots and significant effects were noted. Pair-wise treatment comparison were made for main plot, subplot and mainplot*subplot interaction using Tukey's HSD test. All the treatment effects were compared at $p<0.05$ level.

Table 1. Treatment details of tillage and nutrient management under maize - wheat cropping system

\begin{tabular}{ll}
\hline Treatments & Description \\
\hline Main plot treatment (Tillage) & \\
Bed planting (BP) & For maize and for succeeding wheat \\
Conventional Tillage (CT) & For maize and for succeeding wheat \\
Subplot treatments (manures / fertilizers) & \\
$\mathrm{T}_{1}$ & No N \\
$\mathrm{T}_{2}(100 \% \mathrm{~N})^{\mathrm{a}}$ & $120 \mathrm{~kg}$ urea $-\mathrm{N} / \mathrm{ha}$ \\
$\mathrm{T}_{3}$ & $90 \mathrm{~kg}$ urea-N $+30 \mathrm{~kg} \mathrm{~N} / \mathrm{ha}$ applied \\
& through FYM \\
$\mathrm{T}_{4}$ & $90 \mathrm{~kg}$ urea-N $+30 \mathrm{~kg} \mathrm{~N} / \mathrm{ha}$ applied \\
& through sewage sludge \\
$\mathrm{T}_{5}$ & $120 \mathrm{~kg}$ urea-N/ha $+\mathrm{Crop}$ residues \\
& $($ previous crop) \\
$\mathrm{T}_{6}$ & $120 \mathrm{~kg} \mathrm{~N} / \mathrm{ha}(50 \% \mathrm{FYM}+25 \%$ Bio \\
& fertilizer $+25 \%$ Crop residue $)$ \\
\hline
\end{tabular}

All the treatments have been receiving recommended dose of $\mathrm{P}\left(26.2 \mathrm{~kg} \mathrm{ha}^{-1}\right)$ and $\mathrm{K}\left(50 \mathrm{~kg} \mathrm{ha}^{-1}\right)$ through mineral fertilizers except $\mathrm{T}_{6} \cdot{ }^{\mathrm{a}} 100 \%$ of the recommended dose of $\mathrm{N}$.

\section{Results}

\section{1. Mean weight diameter (MWD)}

MWD was used as a measure of aggregate stability. MWD of soil was affected significantly by nutrient management treatments, while the effect of tillage and their interaction with nutrients was non-significant (Table 2). Plots receiving mineral fertilizers alone $\left(\mathrm{T}_{2}\right)$, mineral fertilizer + organic matter $\left(\mathrm{T}_{3}, \mathrm{~T}_{4}\right.$ and $\left.\mathrm{T}_{5}\right)$ and $100 \%$ organic treatment $\left(\mathrm{T}_{6}\right)$ produced MWD that were statistically at par; and these treatments were found to increase MWD of soil significantly over control $\left(\mathrm{T}_{1}\right)$. In statistically at par; and these treatments were found to increase MWD of soil significantly over control $\left(\mathrm{T}_{1}\right)$. In bed planting, the highest MWD $(1.01 \mathrm{~mm})$ was found in plots receiving $100 \%$ organics $\left(\mathrm{T}_{6}\right)$ whereas in conventional tillage the maximum value $(0.95 \mathrm{~mm})$ was observed in treatment which added crop residues of previous crop to soil $\left(\mathrm{T}_{5}\right)$. The bed planting system showed a good correlation between MWD and SOC, but the effect wasn't that pronounced in conventional tillage (Figure $1 \& 2$ ). 
Table 2. Effect of tillage and nutrient management practices on mean weight diameter ( $\mathrm{mm}$ ) of soil under maize wheat cropping system

\begin{tabular}{|l|c|c|c|}
\hline Treatments & \multicolumn{2}{|c|}{ Tillage (Main plot) } & \multirow{2}{*}{ Mean } \\
\cline { 1 - 2 } $\begin{array}{l}\text { Nutrient management } \\
\text { (Sub plot) }\end{array}$ & BP & CT & \\
\hline $\mathrm{T}_{1}($ Control) & $0.66(0.04)$ & $0.65(0.01)$ & $0.66^{\mathrm{B}}$ \\
\hline $\mathrm{T}_{2}(100 \% \mathrm{~N}$ through urea) & $0.79(0.15)$ & $0.80(0.03)$ & $0.79^{\mathrm{A}}$ \\
\hline $\mathrm{T}_{3}(100 \% \mathrm{~N}$ through urea and FYM) & $0.92(0.13)$ & $0.88(0.02)$ & $0.90^{\mathrm{A}}$ \\
\hline $\mathrm{T}_{4}(100 \% \mathrm{~N}$ through urea and sewage sludge $)$ & $0.94(0.05)$ & $0.91(0.01)$ & $0.93^{\mathrm{A}}$ \\
\hline $\mathrm{T}_{5}(100 \% \mathrm{~N}$ through urea + Crop residues) & $0.98(0.02)$ & $0.95(0.14)$ & $0.97^{\mathrm{A}}$ \\
\hline \multicolumn{1}{|c|}{ Mean } & $1.01(0.07)$ & $0.92(0.01)$ & $0.96^{\mathrm{A}}$ \\
\hline \multicolumn{1}{|c|}{$\mathrm{T} \times \mathrm{N}$} & 0.88 & 0.85 & \\
\hline & \multicolumn{3}{|c|}{$\mathrm{NS}$} \\
\hline
\end{tabular}

${ }^{a}$ Standard deviations are in brackets ${ }^{b}$ Different letters in a column indicate significant differences between the treatments $(p<$ 0.05) BP - Bed planting; CT - Conventional Tillage; NS - Non significant

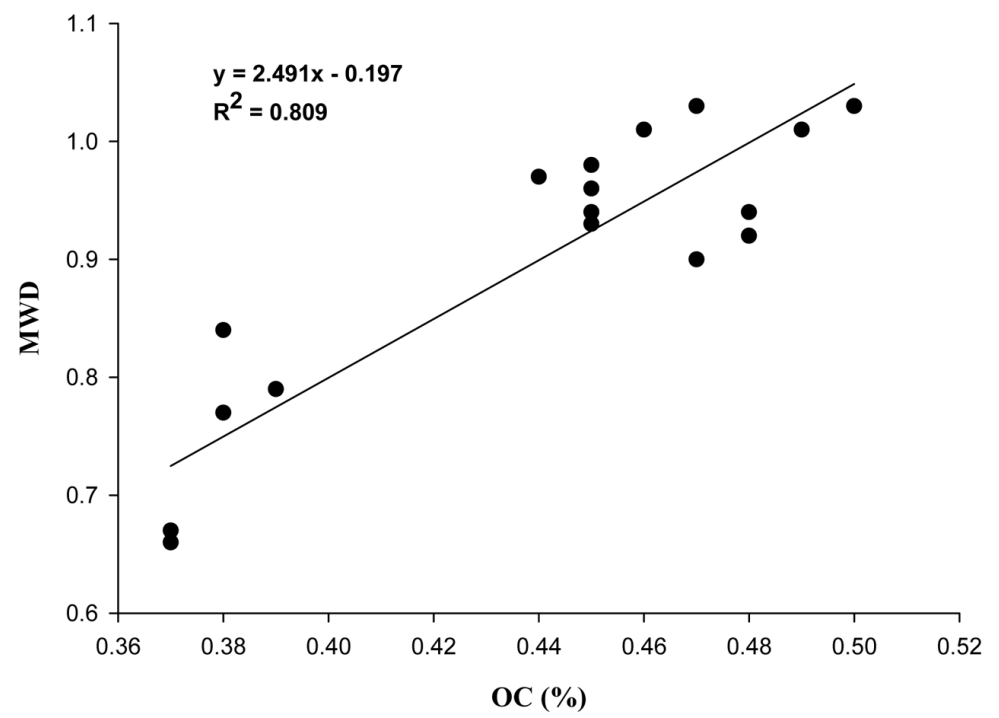

Figure 1. Correlation between organic carbon (\%) and MWD (mm) of soil in maize - wheat system (bed planting) 


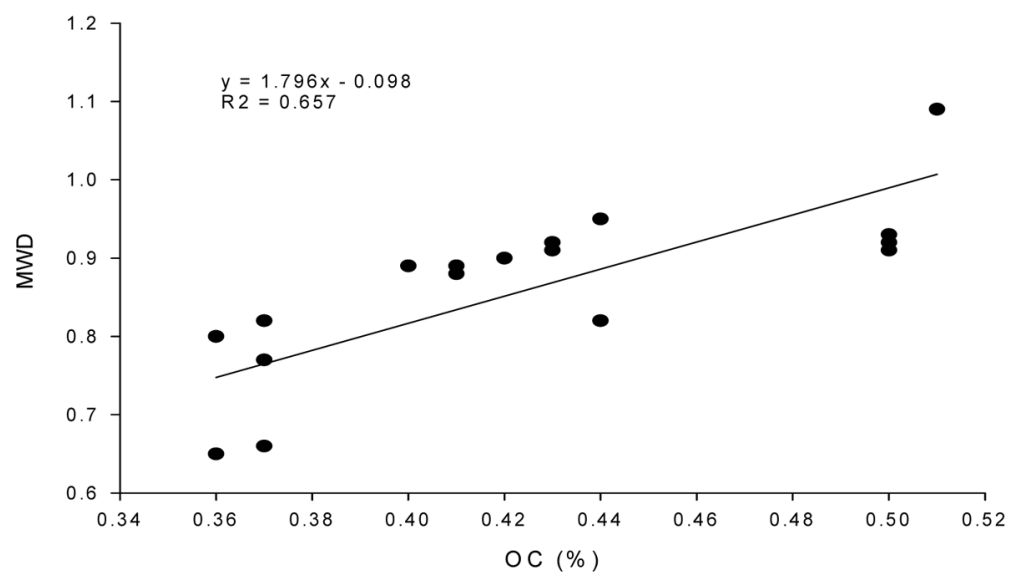

Figure 2. Correlation between organic carbon (\%) and MWD ( $\mathrm{mm}$ ) of soil in maize - wheat system (conventional tillage)

\subsection{Thermal stability of carbon in macroaggregates}

The activation energy and rate constants provide a good insight into decomposability of organic matter and the pace of mineralization in soil aggregates (Table $3 \& 5$ ). The reaction rates of carbon decomposition were significantly lower in bed planting than conventional tillage system. Macroaggregates from $\mathrm{T}_{2}(100 \% \mathrm{~N}+\mathrm{PK})$ in bed planting showed a gradual increase in reaction rate with temperature and the rates ranged from 1.17 to $2.08 \mathrm{mmols} \mathrm{C} / \mathrm{day}$. In bed planting system, plots receiving FYM $\left(\mathrm{T}_{3}\right)$ showed a rapid increase in soil carbon decomposition between $25^{\circ} \mathrm{C}$ and 30 ${ }^{\circ} \mathrm{C}$ (from 1.00 to $1.50 \mathrm{mmols} \mathrm{C} / \mathrm{day}$ ) and a gradual change thereafter. A rapid change in the reaction rates (k values) from 1.83 to 2.58 and 2.00 to 2.75 mmols $\mathrm{C} /$ day was observed in macroaggregates of bed planting plots treated with crop residues $\left(\mathrm{T}_{5}\right)$ and $100 \%$ organics $\left(\mathrm{T}_{6}\right)$ respectively, between temperatures $35{ }^{\circ} \mathrm{C}$ and $40{ }^{\circ} \mathrm{C}$ indicating a higher temperature sensitivity for SOC in these plots. The first order rate kinetics in conventional tillage were significantly higher than their corresponding treatments in bed planting indicating a lower stability of carbon in the former system. In conventional tillage system $\mathrm{k}$ values ranged between $1.42 \mathrm{mmols} \mathrm{C} /$ day $\left(\mathrm{T}_{2}\right.$ at $\left.25^{\circ} \mathrm{C}\right)$ and $3.75 \mathrm{mmols} \mathrm{C} /$ day $\left(\mathrm{T}_{5}\right.$ at $\left.40^{\circ} \mathrm{C}\right)$. No significant interaction of tillage, nutrient management and reaction temperatures on carbon decomposition was found in macroaggregates of the maize-wheat system.

Activation energies for soil organic carbon decomposition in macroaggregates was found to be maximum in $100 \%$ organics treated plots $\left(\mathrm{T}_{6}\right)$ and minimum in the control plots $\left(\mathrm{T}_{1}\right)$. Activation energy indicates thermal sensitivity of organic carbon.

More the activation energy, more is the thermal sensitivity and vice versa. Among the plots with integrated nutrient application $\left(\mathrm{T}_{3}\right.$ to $\left.\mathrm{T}_{5}\right), \mathrm{FYM}\left(\mathrm{T}_{3}\right)$ and crop residue $\left(\mathrm{T}_{3}\right)$ treatments had comparable activation energies which were significantly lower than other organic treated ones $\left(\mathrm{T}_{4}\right.$ and $\left.\mathrm{T}_{6}\right)$ indicating the lower temperature sensitivity of SOC in these plots. 
Table 3. First order rate constants (k) of carbon decomposition at different temperatures in soil macroaggregates of bed planting and conventional tillage systems

\begin{tabular}{|c|c|c|c|c|c|c|c|c|c|c|}
\hline \multirow{3}{*}{$\begin{array}{c}\text { Nutrient } \\
\text { management } \\
\text { (Sub Plot) }\end{array}$} & \multicolumn{5}{|c|}{ Bed Planting } & \multicolumn{5}{|c|}{ Conventional Tillage } \\
\hline & \multicolumn{4}{|c|}{$\begin{array}{l}\text { Rate constant (k) in mmols } \\
\text { C/ day at }\end{array}$} & \multirow[t]{2}{*}{ Mean } & \multicolumn{4}{|c|}{$\begin{array}{l}\text { Rate constant (k) in mmols } \\
\text { C/ day at }\end{array}$} & \multirow[t]{2}{*}{ Mean } \\
\hline & $25^{\circ} \mathrm{C}$ & $30^{\circ} \mathrm{C}$ & $35^{\circ} \mathrm{C}$ & $40^{\circ} \mathrm{C}$ & & $25^{\circ} \mathrm{C}$ & $30^{\circ} \mathrm{C}$ & $35^{\circ} \mathrm{C}$ & $40^{\circ} \mathrm{C}$ & \\
\hline $\mathrm{T}_{1}$ ( Control) & $\begin{array}{c}1.58 \\
(0.01)\end{array}$ & $\begin{array}{c}1.58 \\
(0.02)\end{array}$ & $\begin{array}{c}1.58 \\
(0.03)\end{array}$ & $\begin{array}{c}2.33 \\
(0.10) \\
\end{array}$ & 1.77 & $\begin{array}{c}1.83 \\
(0.08)\end{array}$ & $\begin{array}{c}1.75 \\
(0.02)\end{array}$ & $\begin{array}{c}1.67 \\
(0.01)\end{array}$ & $\begin{array}{c}2.58 \\
(0.11)\end{array}$ & 1.96 \\
\hline $\begin{array}{l}\mathrm{T}_{2}(100 \% \mathrm{~N} \\
\text { through urea) }\end{array}$ & $\begin{array}{c}1.17 \\
(0.06)\end{array}$ & $\begin{array}{c}1.17 \\
(0.04)\end{array}$ & $\begin{array}{c}1.33 \\
(0.02)\end{array}$ & $\begin{array}{c}2.08 \\
(0.17) \\
\end{array}$ & 1.44 & $\begin{array}{c}1.42 \\
(0.11)\end{array}$ & $\begin{array}{c}1.33 \\
(0.05)\end{array}$ & $\begin{array}{c}1.33 \\
(0.06)\end{array}$ & $\begin{array}{c}2.17 \\
(0.18)\end{array}$ & 1.56 \\
\hline $\begin{array}{l}\mathrm{T}_{3}(100 \% \mathrm{~N} \\
\text { through urea } \\
\text { and FYM })\end{array}$ & $\begin{array}{l}1.00 \\
(0.02)\end{array}$ & $\begin{array}{l}1.50 \\
(0.03)\end{array}$ & $\begin{array}{l}1.58 \\
(0.04)\end{array}$ & $\begin{array}{c}2.00 \\
(0.08)\end{array}$ & 1.52 & $\begin{array}{l}1.50 \\
(0.06)\end{array}$ & $\begin{array}{c}1.58 \\
(0.03)\end{array}$ & $\begin{array}{c}1.67 \\
(0.03)\end{array}$ & $\begin{array}{c}2.50 \\
(0.10)\end{array}$ & 1.81 \\
\hline $\begin{array}{l}\mathrm{T}_{4}(100 \% \mathrm{~N} \\
\text { through urea } \\
\text { and sewage } \\
\text { sludge })\end{array}$ & $\begin{array}{c}1.17 \\
(0.03)\end{array}$ & $\begin{array}{c}1.33 \\
(0.02)\end{array}$ & $\begin{array}{l}1.92 \\
(0.14)\end{array}$ & $\begin{array}{c}2.33 \\
(0.24)\end{array}$ & 1.69 & $\begin{array}{c}1.75 \\
(0.18)\end{array}$ & $\begin{array}{c}1.92 \\
(0.02)\end{array}$ & $\begin{array}{l}2.00 \\
(0.04)\end{array}$ & $\begin{array}{c}3.00 \\
(0.30)\end{array}$ & 2.17 \\
\hline $\begin{array}{l}\mathrm{T}_{5}(100 \% \mathrm{~N} \\
\text { through urea }+ \\
\text { Crop residues })\end{array}$ & $\begin{array}{c}1.25 \\
(0.03)\end{array}$ & $\begin{array}{c}1.67 \\
(0.24)\end{array}$ & $\begin{array}{c}1.83 \\
(0.18)\end{array}$ & $\begin{array}{c}2.58 \\
(0.19)\end{array}$ & 1.83 & $\begin{array}{c}2.42 \\
(0.17)\end{array}$ & $\begin{array}{c}2.42 \\
(0.35)\end{array}$ & $\begin{array}{c}2.67 \\
(0.07)\end{array}$ & $\begin{array}{c}3.75 \\
(0.27)\end{array}$ & 2.81 \\
\hline $\begin{array}{l}\mathrm{T}_{6}(100 \% \\
\text { organics) }\end{array}$ & $\begin{array}{c}1.08 \\
(0.03)\end{array}$ & $\begin{array}{c}1.67 \\
(0.02)\end{array}$ & $\begin{array}{c}2.00 \\
(0.10)\end{array}$ & $\begin{array}{c}2.75 \\
(0.22) \\
\end{array}$ & 1.87 & $\begin{array}{c}1.58 \\
(0.13)\end{array}$ & $\begin{array}{c}1.75 \\
(0.02)\end{array}$ & $\begin{array}{c}1.92 \\
(0.02)\end{array}$ & $\begin{array}{c}2.92 \\
(0.23)\end{array}$ & 2.04 \\
\hline Mean & 1.21 & 1.49 & 1.71 & 2.35 & $1.69^{B}$ & 1.75 & 1.79 & 1.87 & 2.82 & $2.06^{A}$ \\
\hline
\end{tabular}

${ }^{a}$ Standard deviations are in brackets ${ }^{\mathrm{b}} \mathbf{C D}(<\mathbf{0 . 0 5})$ : $\mathbf{T}$ means -0.08 ; $\mathbf{T}$ means at same or different level of $\mathbf{N}-0.12$; $\mathbf{T}$ Means at same or different level of R- 0.10; T Means at same or different level of $\mathbf{N} \times \mathbf{R}-\mathrm{NS} ; \quad \mathbf{N}$ Means at same or different level of $\mathbf{T}-0.11 ; \mathbf{R}$ Means at same or different level of $\mathbf{T}-0.09 ; \quad \mathbf{N} \times \mathbf{R}$ Means at same or different level of $\mathbf{T}-\mathrm{NS} ; \mathbf{R}$ Means at same or different level of $\mathbf{T} \times \mathbf{N}$ - NS; $\mathbf{N}$ Means a same or different level of $\mathbf{T} \times \mathbf{R}$ - NS. T- Tillage; $\mathbf{N}$ - nutrient management; $\mathbf{R}$ - Reaction rate at a particular temperature; NS - Non significant. 'Different letters in a row indicate significant differences between the treatments $(p<0.05)$.

\subsection{Thermal stability of carbon in microaggregates}

It has been shown that microaggregates provide better protection to organic carbon by a greater complexation mechanism compared to macroaggregates (Six et al. 2002, 2004). In agreement with the literature ,the study shows that decomposition rate of carbon stored in microaggregates, as indicated by reaction rates, were lower than corresponding values for macroaggregates in both tillage systems (Table 4). Among organic manure treated plots in conventional tillage system, microaggregates from plots receiving mineral fertilizers + FYM $\left(\mathrm{T}_{3}\right)$ had significantly higher reaction rates of carbon decomposition, while plots treated with residues from previous crop $\left(\mathrm{T}_{5}\right)$ and $100 \%$ organics $\left(\mathrm{T}_{6}\right)$ had the highest stability of carbon (lowest first order reaction rates). Though the rate constant values were higher in $\mathrm{T}_{6}$ (100\% organics) of bed planting system, high activation energy $\left(59.8 \mathrm{kJmol}^{-1}\right)$ ensures that a substantial amount of aggregate organic carbon is protected from decomposition until the requisite energy levels are provided (Table 5). The activation energy for decomposition of organic carbon in microaggregates ranged from 23.5 to $134.2 \mathrm{kJmol}^{-1}$ in bed planting system and varied between 22.0 to 53.8 $\mathrm{kJmol}^{-1}$ in conventional tillage. Under conventional tillage system, microaggregates from $T_{1}$ (plots receiving no treatment) and $T_{2}$ (mineral fertilizers alone) indicated the most vulnerable organic carbon as their activation energies $\left(24.3 \mathrm{kJmol}^{-1}\right.$ and 23.5 $\mathrm{kJmol}^{-1}$, respectively) were significantly lower than all other treatments. Carbon in microaggregates 
of bed planting + crop residues $\left(\mathrm{T}_{5}\right)$ needed 134.2 $\mathrm{kJmol}^{-1}$ to initiate and further residues $\left(\mathrm{T}_{5}\right)$ needed $134.2 \mathrm{kJmol}^{-1}$ to initiate and further a decomposition reaction, a value much higher than that of any other treatment combination in the study. The $\mathrm{k}$ values of carbon decomposition in $\mathrm{T}_{6}(100 \%$ organics $)$ was only half of that in other treatments at all temperatures, except at $40{ }^{\circ} \mathrm{C}$.

Table 5. Activation energy $\left(\mathrm{kJmol}^{-1}\right)$ of carbon decomposition in soil macro and microaggregates of bed planting and conventional tillage systems

\begin{tabular}{|c|c|c|c|c|c|c|}
\hline \multirow{2}{*}{$\begin{array}{l}\text { Nutrient management } \\
\text { (Sub Plot) }\end{array}$} & \multicolumn{3}{|c|}{ Macroaggregates } & \multicolumn{3}{|c|}{ Microaggregates } \\
\hline & BP & CT & Mean & BP & CT & Mean \\
\hline $\mathrm{T}_{1}($ Control $)$ & 19.8 & 14.7 & $17.3^{\mathrm{D}}$ & 24.3 & 24.5 & $24.4^{\mathrm{E}}$ \\
\hline $\mathrm{T}_{2}(100 \% \mathrm{~N}$ through urea) & 31.9 & 19.3 & $25.6^{\mathrm{C}}$ & 23.5 & 22 & $22.8^{\mathrm{E}}$ \\
\hline $\begin{array}{l}\mathrm{T}_{3}(100 \% \mathrm{~N} \text { through urea and } \\
\mathrm{FYM})\end{array}$ & 33.9 & 24.8 & $29.4^{\mathrm{CB}}$ & 56.6 & 31.2 & $43.9^{C}$ \\
\hline $\begin{array}{l}\mathrm{T}_{4}(100 \% \mathrm{~N} \text { through urea and } \\
\text { sewage sludge })\end{array}$ & 38.6 & 26.1 & $32.4^{\mathrm{B}}$ & 39.9 & 34.3 & $37.1^{\mathrm{D}}$ \\
\hline $\begin{array}{l}\mathrm{T}_{5}(100 \% \mathrm{~N} \text { through urea }+ \\
\text { Crop residues })\end{array}$ & 35.4 & 21.7 & $28.6^{\mathrm{CB}}$ & 134.2 & 34.3 & $84.3^{\mathrm{A}}$ \\
\hline $\mathrm{T}_{6}(100 \%$ organics $)$ & 48.9 & 28.6 & $38.8^{\mathbf{A}}$ & 59.8 & 53.8 & $56.8^{\mathrm{B}}$ \\
\hline Mean & $34.8^{\mathrm{a}}$ & $22.5^{b}$ & & $56.4^{\mathrm{a}}$ & $33.4^{b}$ & \\
\hline $\mathbf{T} \times \mathbf{N}$ & \multicolumn{3}{|c|}{ NS } & \multicolumn{3}{|c|}{$16.9(\mathrm{x}) ; 16.8(\mathrm{y})$} \\
\hline
\end{tabular}

${ }^{a}$ Different capital letters in a column indicate significant differences between the treatments $(p<0.05)$. ${ }^{b}$ Different small letters in a row indicate significant differences between the treatments $(p<0.05)$. ${ }^{\mathrm{c}} \mathrm{BP}-\mathrm{Bed}$ planting; $\mathrm{CT}$ - Conventional Tillage; NS - Non significant. ${ }^{d} \mathrm{X}=$ For the same level of main plot treatment; $\mathrm{y}=$ For the same level of sub plot treatment

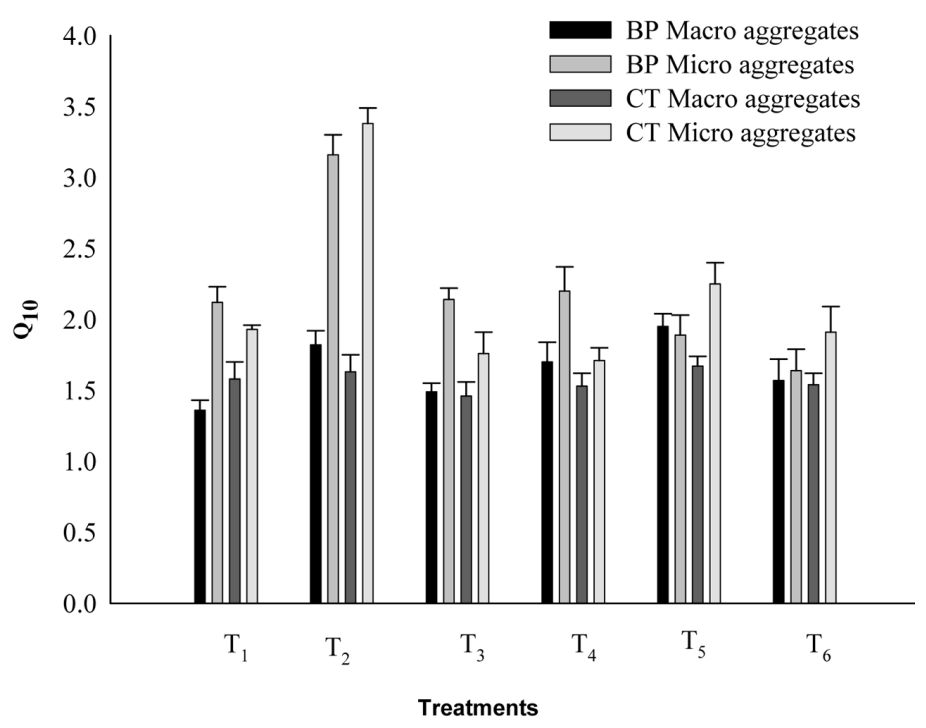

Figure 3. Effect of tillage and nutrient management on $\mathrm{Q}_{10}$ values of macro and microaggregates 
Table 4. First order rate constants $(\mathrm{k})$ of carbon decomposition at different temperatures in soil microaggregates of bed planting and conventional tillage systems

\begin{tabular}{|c|c|c|c|c|c|c|c|c|c|c|}
\hline \multirow{3}{*}{$\begin{array}{c}\text { Nutrient } \\
\text { management } \\
\text { (Sub Plot) }\end{array}$} & \multicolumn{5}{|c|}{ Bed Planting } & \multicolumn{5}{|c|}{ Conventional Tillage } \\
\hline & \multicolumn{4}{|c|}{$\begin{array}{l}\text { Rate constant (k) in mmols } \\
\text { C/ day at }\end{array}$} & \multirow[t]{2}{*}{ Mean } & \multicolumn{4}{|c|}{$\begin{array}{l}\text { Rate constant (k) in mmols } \\
\text { C/ day at }\end{array}$} & \multirow[t]{2}{*}{ Mean } \\
\hline & $25^{\circ} \mathrm{C}$ & $3^{\circ}{ }^{\circ} \mathrm{C}$ & $35^{\circ} \mathrm{C}$ & $40^{\circ} \mathrm{C}$ & & $25^{\circ} \mathrm{C}$ & $30^{\circ} \mathrm{C}$ & $35^{\circ} \mathrm{C}$ & $40^{\circ} \mathrm{C}$ & \\
\hline $\mathrm{T}_{1}$ ( Control) & $\begin{array}{c}0.83 \\
(0.01)\end{array}$ & $\begin{array}{c}0.75 \\
(0.03) \\
\end{array}$ & $\begin{array}{c}0.58 \\
(0.00) \\
\end{array}$ & $\begin{array}{c}1.50 \\
(0.03)\end{array}$ & 0.92 & $\begin{array}{c}1.25 \\
(0.02)\end{array}$ & $\begin{array}{c}1.08 \\
(0.05) \\
\end{array}$ & $\begin{array}{c}1.17 \\
(0.01)\end{array}$ & $\begin{array}{c}2.08 \\
(0.03) \\
\end{array}$ & 1.40 \\
\hline $\begin{array}{l}\mathrm{T}_{2}(100 \% \mathrm{~N} \\
\text { through urea) }\end{array}$ & $\begin{array}{c}0.67 \\
(0.02)\end{array}$ & $\begin{array}{c}0.58 \\
(0.05)\end{array}$ & $\begin{array}{c}0.92 \\
(0.05) \\
\end{array}$ & $\begin{array}{c}1.83 \\
(0.03)\end{array}$ & 1.00 & $\begin{array}{c}1.25 \\
(0.02)\end{array}$ & $\begin{array}{c}1.25 \\
(0.10) \\
\end{array}$ & $\begin{array}{c}1.00 \\
(0.05)\end{array}$ & $\begin{array}{c}2.17 \\
(0.08)\end{array}$ & 1.42 \\
\hline $\begin{array}{l}\mathrm{T}_{3}(100 \% \mathrm{~N} \\
\text { through urea } \\
\text { and FYM })\end{array}$ & $\begin{array}{c}0.58 \\
(0.01)\end{array}$ & $\begin{array}{c}0.92 \\
(0.04)\end{array}$ & $\begin{array}{c}0.92 \\
(0.00)\end{array}$ & $\begin{array}{l}2.00 \\
(0.05)\end{array}$ & 1.10 & $\begin{array}{l}1.58 \\
(0.04)\end{array}$ & $\begin{array}{c}1.58 \\
(0.06)\end{array}$ & $\begin{array}{l}1.75 \\
(0.03)\end{array}$ & $\begin{array}{l}3.08 \\
(0.06)\end{array}$ & 2.00 \\
\hline $\begin{array}{l}\mathrm{T}_{4}(100 \% \mathrm{~N} \\
\text { through urea } \\
\text { and sewage } \\
\text { sludge })\end{array}$ & $\begin{array}{c}0.83 \\
(0.01)\end{array}$ & $\begin{array}{c}0.83 \\
(0.08)\end{array}$ & $\begin{array}{c}1.00 \\
(0.00)\end{array}$ & $\begin{array}{l}1.83 \\
(0.14)\end{array}$ & 1.12 & $\begin{array}{l}1.58 \\
(0.12)\end{array}$ & $\begin{array}{c}1.50 \\
(0.15)\end{array}$ & $\begin{array}{l}1.25 \\
(0.03)\end{array}$ & $\begin{array}{c}2.50 \\
(0.03)\end{array}$ & 1.71 \\
\hline $\begin{array}{l}\mathrm{T}_{5}(100 \% \mathrm{~N} \\
\text { through urea }+ \\
\text { Crop residues })\end{array}$ & $\begin{array}{c}0.17 \\
(0.02)\end{array}$ & $\begin{array}{c}1.17 \\
(0.08)\end{array}$ & $\begin{array}{c}1.17 \\
(0.00)\end{array}$ & $\begin{array}{l}2.25 \\
(0.22)\end{array}$ & 1.19 & $\begin{array}{c}1.17 \\
(0.11)\end{array}$ & $\begin{array}{c}1.08 \\
(0.08)\end{array}$ & $\begin{array}{c}1.25 \\
(0.03)\end{array}$ & $\begin{array}{c}2.42 \\
(0.35)\end{array}$ & 1.48 \\
\hline $\begin{array}{l}\mathrm{T}_{6}(100 \% \\
\text { organics })\end{array}$ & $\begin{array}{c}1.50 \\
(0.02)\end{array}$ & $\begin{array}{c}1.50 \\
(0.12)\end{array}$ & $\begin{array}{c}1.50 \\
(0.05) \\
\end{array}$ & $\begin{array}{c}2.50 \\
(0.13)\end{array}$ & 1.75 & $\begin{array}{c}0.58 \\
(0.03)\end{array}$ & $\begin{array}{c}0.58 \\
(0.04) \\
\end{array}$ & $\begin{array}{c}0.58 \\
(0.01)\end{array}$ & $\begin{array}{c}1.92 \\
(0.02)\end{array}$ & 0.92 \\
\hline Mean & 0.76 & 0.96 & 1.01 & 1.99 & $1.18^{B}$ & 1.02 & 1.01 & 1.00 & 2.02 & $1.49^{A}$ \\
\hline
\end{tabular}

${ }^{a}$ Standard deviations are in brackets. ${ }^{\mathrm{b}} \mathrm{CD}(<0.05)$ : $\mathbf{T}$ means $-0.05 ; \mathbf{T}$ means at same or different level of $\mathbf{N}-0.08$; $\mathbf{T}$ Means at same or different level of R- 0.07; $\mathbf{T}$ Means at same or different level of $\mathbf{N} \times \mathbf{R}-0.14 ; \quad \mathbf{N}$ Means at same or different level of T - 0.07; R Means at same or different level of T - 0.06; $\mathbf{N} \times \mathbf{R}$ Means at same or different level of $\mathbf{T}-0.14 ; \mathbf{R}$ Means at same or different level of $\mathbf{T} \times \mathbf{N}-0.14 ; \mathbf{N}$ Means at same or different level of $\mathbf{T} \times \mathbf{R}-0.14$. T- Tillage; $\mathbf{N}$ - nutrient management; $\mathbf{R}$ - Reaction rate at a particular temperature. ${ }^{c}$ Different letters in a row indicate significant differences between the treatments $(p<0.05)$.

\section{4. $Q_{10}$ values of carbon decomposition in macro and microaggregates}

Equation 3 depicts that if rate of the reaction is completely temperature independent, the resulting $\mathrm{Q}_{10}$ will be 1.0 and the value increases with increasing thermal dependency of the reaction. Here we used $\mathrm{Q}_{10}$ as an indicator of temperature dependencies of carbon decomposition in soil aggregates and also to infer the reaction rate changes over a $10^{\circ} \mathrm{C}$ range of temperature rise. The temperature control on carbon decomposition was found to be more important in macroaggregates derived from bed planting than conventional tillage system in all the nutrient treatments, except for $\mathrm{T}_{1}$ (control) and $\mathrm{T}_{3}$ (NPK +FYM) (Figure 3). In macroaggregates of $\mathrm{T}_{1}$ and $\mathrm{T}_{3}, \mathrm{Q}_{10}$ values were found to be around 1.5 indicating lesser temperature dependency of carbon decomposition. Microaggregates gave higher $\mathrm{Q}_{10}$ values than macroaggregates in all the treatments and the highest variation was found in $T_{2}$. High thermal sensitivity of carbon decomposition in microaggregates of $T_{2}$ (mineral fertilizers alone) was evident from the $\mathrm{Q}_{10}$ values of $\sim 4$ in these treatments.

\section{Discussion}

\subsection{Mean weight diameter of soils}

Water stable aggregates of the maize - wheat system was affected significantly by nutrient management treatments $\left(\mathrm{T}_{2}\right.$ to $\left.\mathrm{T}_{6}\right)$ which gave higher MWD than control $\left(T_{1}\right)$. Plots receiving manures and fertilizers 
essentially add organic carbon to the soil either by way of treatment itself or by improving crop growth/ biomass. In both bed planting $\left(\mathrm{r}^{2}=0.858\right)$ and conventional tillage $\left(r^{2}=0.621\right)$ a close relationship was obtained between MWD and SOC. SOC forms complexes with primary mineral particles and secondary structural units leading to aggregates with high water stability. Earlier works of Munkholm (2011) also prove the complementary interaction between SOC and MWD. Unlike nutrient management, tillage treatments were found to have no effect on MWD of soils. This suggests a higher influence of nutrient management compared to tillage on water stability of aggregates or a masking of tillage effects by nutrient management. Obliteration of the effects of tillage on soil physical properties by manurial application has also been reported by Bauer and Black (1981) and Lal (1999).

\subsection{Thermal stability of carbon in macroaggregates}

The study showed that all nutrient treatments in conventional tillage had comparatively higher rate constant values than corresponding treatments of bed planting at all study temperatures. Conventional tillage with greater soil disturbance disrupted aggregates and led to enhanced aggregate turnover and decomposition of SOM (Paustian et al. 1998; Six et al. 1998). A similar hypothesis put forward by Elliot (1986) was that macroaggregates contain more labile and less highly processed SOM and that this organic matter is lost on continuous disturbance. Some authors also argue that macroaggregation, as a transitional property of soil, would exert only a minimal physical protection, the macroaggregates destroying and reforming themselves constantly, unlike stable microaggregates (Six et al. 2004; Kong et al. 2005). On the other hand, bed planting system with its lesser soil disturbance generates an in situ temperature insensitive process that alters the release of easily decomposable substrates and reduces the decomposition rate. Moreover in bed planting system, addition of organic carbon $\left(\mathrm{T}_{3}\right.$ to $\mathrm{T}_{6}$ ) enhances the aggregation process and provides a physical barrier to decomposition factors (microbes and extracellular enzymes). Conventional tillage with its lesser support for aggregation couldn't generate the same effect even with addition of organic matter. We observed an increase in carbon decomposition rates with temperature indicating a thermal sensitivity for stable carbon substrate. Boddy et al. (2008) and Conen et al. (2006) had reported earlier that when available substrates are depleted and no other external constraints restrict decomposition, the soil microflora can enzymatically acclimate to decompose stable SOM, which reveals a potentially higher sensitivity of these fractions than labile SOM at higher temperatures. In the present study, we have used both activation energy Ea and $Q_{10}$ to draw mechanistic information about the SOC decomposition process. Among different nutrient managements, it was found that the activation energies were maximum in $100 \%$ organics treated plots $\left(\mathrm{T}_{6}\right)$ and lowest in control plots $\left(\mathrm{T}_{1}\right)$ in both tillage systems. However, this cannot be construed as an indication of resistant organic carbon build up in the former treatment, but may be just due to exclusion of decomposition enzymes from OC substrates within the aggregate environment, causing substrate limitation at reaction microsites (Davidson et al., 2006). The $\mathrm{Q}_{10}$ values $\approx 2$ for $\mathrm{T}_{6}$ indicates the higher temperature sensitivity and higher substrate availability of SOC in these treatments with temperature rises. Increases in $\mathrm{Q}_{10}$ values as a direct effect of substrate availability was also reported by Gershenson et al. (2009). $\mathrm{T}_{3}$ (FYM treatment) and $\mathrm{T}_{5}$ (crop residue treatment) had lower activation energies of SOC decomposition among the organic treated plots indicating a lesser thermal sensitivity for macroaggregate carbon. However a perusal of the $\mathrm{Q}_{10}$ values shows that SOC in macroaggregates of $T_{5}\left(Q_{10}>2\right)$ is highly temperature responsive and the higher activation energy is due to chemical recalcitrance of the residues which is overcome with rises in temperature. At higher temperatures the decomposition barrier of crop residues is overcome either due to reduced $\mathrm{C}: \mathrm{N}$ ratio (higher $\mathrm{C}$ decomposition) or by an increased enzyme activity. $Q_{10}$ values of $T_{5}$ in conventional tillage (1.67) was lower than bed planting (1.95) as the crop residues 
are made vulnerable more easily in the former treatment due to its higher soil disturbance thereby negating the temperature effect on decomposition to some effect. So it can be deducted that the higher activation energies reported in $T_{5}$ is an effect of chemical recalcitrance of crop residues rather than an aggregate mediated physical protection of carbon. Applying the same logic, low $\mathrm{Q}_{10}$ values of $\mathrm{T}_{3}(1.49 \mathrm{in}$ bed planting and $1.46 \mathrm{in}$ conventional tillage systems), among the treatments studied, along with its promotion of aggregate stability (MWD values of 0.92 $\mathrm{mm}$ in bed planting and $0.88 \mathrm{~mm}$ in conventional tillage) shows that this integrated nutrient management with mineral fertilizer + FYM is an option to promote physical protection of applied SOC in soil.

SOC in macroaggregates of mineral fertilizer + FYM treatment $\left(\mathrm{T}_{3}\right)$ can be considered the most resistant among the different treatments as indicated by its very low $\mathrm{Q}_{10}$ values.

There was a jump in reaction rates with temperature in bed planting ( $\mathrm{T}_{1}$ to $\mathrm{T}_{6}$ ) whereas the decomposition rate change was more or less gradual in conventional tillage. The rate of a reaction is dependent on substrate/ reactant availability. In aggregates of bed planting system, there is a lumping of protected simple compounds and more complex unprotected compounds together into a common pool which are exposed to decomposition forces only at higher temperatures either by faster desorption of carbon from the adsorption sites (Hulscher and Cornelissen, 1996) or by increased efficacy of biological decomposition processes at these temperatures (Reichstein et al., 2005). On the contrary, in conventional tillage system frequent soil disturbances regularly break carbon rich macroaggregates and ensure a steady supply of carbon substrates for decomposition. It has been widely accepted that sensitivity to temperature changes must be evaluated within the context of substrate availability (Imriskova et al., 2005). However, the conditions of changing substrate availability may limit applicability of Arrhenius kinetics and can be better explained by models of enzyme catalysed processes such as Michaelis-Menten kinetics (Atkin and Tjoelker, 2003).

\subsection{Thermal stability of carbon in microaggregates}

The decomposition rates of carbon in microaggregates of both tillage systems were lower than their corresponding values in macroaggregate fraction. The lower carbon decomposition rates in microaggregates can be attributed to higher level of carbon protection and thereby effective exclusion of microbes and extracellular enzymes (Von Lützow et al. 2006). Further, micro aggregates produces an in situ anaerobic and resulting reducing conditions thereby restricting microbial activity on the carbon substrates (Six et al., 2000). The strong physico - chemical and physical protection offered by microaggregates to organic carbon effectively increases energy of activation $\left(\mathrm{E}_{\mathrm{a}}\right.$ ) leading to decreased decomposition rates. Under conventional tillage system a disturbance induced microaggregate turnover effectively prevents the same level of carbon protection as that of bed planting. Incorporation of crop residues in bed planting system gave the highest activation energy for carbon decomposition in microaggregates. High C: $\mathrm{N}$ ratio of incorporated residues (rice and wheat) acted as deterrents for faster microbial decomposition of carbon leading to higher activation energies for the reaction. The Arrhenius function shows that temperature sensitivity of decomposition increases with increasing stability of organic compounds. Hence the stable pool can be considered more temperaturesensitive than the labile pool, which is characterised by low activation energies. Apart from this, increases in temperature could accelerate desorption of sorbed carbon from soil minerals (Six et al., 2004) and increase reaction rates and $\mathrm{Q}_{10}$ values. Though the $\mathrm{Q}_{10}$ values of microaggregates were consistently higher than macroaggregates we could not elucidate a one - to - one relationship between activation energy and $\mathrm{Q}_{10}$ in microaggregates with respect to tillage or nutrient management. This paradox in correlating the $\mathrm{Q}_{10}$ and activation energies in some cases appears because both Arrhenius equation and reaction rates demonstrate varying temperature sensitivities of differently stabilized organic pools (Davidson and Janssens, 2006). It should be noted 
that the study handled bulk SOM in aggregates as one homogeneous pool and ignored the variation in relative abundances of differently stabilised SOM pools and their underlying temperature dependencies in decomposition rates. Results are thereby restricted to potential temperature sensitivity of bulk SOM rather than actual temperature sensitivity of each individual pool. Apart from reactivity, decomposition rates under substrate constrained conditions (such as in microaggregates) also depends on the quality and enzyme affinity for individual substrate, which is not considered by Arrhenius equation. The limitation of Arrhenius kinetics under conditions of low substrate availability was also outlined by Von Lützow and Kögel-Knabner (2009).

\section{Conclusion}

Organic carbon acts as a good binding agent for soil particles and any addition of organic inputs to soil will improve aggregation irrespective of the tillage system. The addition of nutrients either through mineral fertilizers or organic manures by way of treatment itself or by improving crop growth/ biomass has the potential to nullify effects of tillage on water stable aggregates in soil. However, an increased soil disturbance by tillage accelerates the SOC decomposition by altering physical protection or reducing chemical recalcitrance of carbon in soil aggregates. Mineral fertilizer + FYM was found to promote aggregation as well as thermal stability of SOC in maize - wheat rotation systems of semi - arid India. Based on our observations, we recommend a treatment combination of reduced tillage and mineral fertilizer + FYM for SOC build up in this major cropping system of the region. We also consider a combination of the Arrhenius equation derived activation energy, $\mathrm{Q}_{10}$ and Michaelis Menten kinetics as effective monitoring parameters of thermal stability of carbon in different cropping systems.

\section{Acknowledgements}

Authors thank the Head, Division of Soil Science and Agricultural Chemistry, Indian Agricultural Research Institute, New Delhi, India for providing all the required facilities to carry out the present investigation.

\section{References}

Aggarwal, P.K., Kalra, N., Chander, S., Pathak, H., 2006. InfoCrop: A dynamic simulation model for the assessment of crop yields, losses due to pests, and environmental impact of agro-ecosystems in tropical environments. I. Model description. Agr Syst. 89, 1- 25.

Ashagrie, Y., Zech, W., Guggenberger, G., Mamo, T., 2007. Soil aggregation, and total and particulate organic matter following conversion of native forests to continuous cultivation in Ethiopia. Soil Till. Res. 94, 101-108.

Atkin, O.K., Tjoelker, M.G. 2003. Thermal acclimation and the dynamic response of plant respiration to temperature. Trends Plant Sci. 8, 343 -351.

Bhattacharyya. T., Pal, D.K., Williams, S., Telpande, B.A., Deshmukh, A.S., Chandran, P., Ray, S.K., Mandal, C., Easter, M., Paustian. K. 2010. Evaluating the Century $\mathrm{C}$ model using two long-term fertilizer trials representing humid and semi-arid sites from India. Agric Ecosyst Environ. 139, 264-272.

Bauer, A., Black, A.L. 1981. Soil carbon, nitrogen, and bulk density comparison in two crop land tillage systems after 25 years in virgin grassland. Soil Sci. Soc. Am. J. 45, 1160-1170.

Boddy, E., Roberts, P., Hill, P.W., Farrar, J., Jones, D.L. 2008. Turnover of low molecular weight dissolved organic $\mathrm{C}$ (DOC) and microbial C exhibit different temperature sensitivities in Arctic tundra soils. Soil Biol. Biochem. 40, 1557-1566. 
Conen, F., Leifeld, J., Seth, B., Alewell, C. 2006. Warming mineralises young and old soil carbon equally. Biogeosciences. 3, 515- 519.

Davidson, E.A., Janssens, I.A. 2006. Temperature sensitivity of soil carbon decomposition and feedbacks to climate change. Nature. 440, 65- 173

Davidson, E.A., Janssens, I.A., Luo, Y. 2006. On the variability of respiration in terrestrial ecosystems: moving beyond $\mathrm{Q}_{10}$. Global Change Biol. 12, 154164.

Elliott, E.T., 1986. Aggregate structure and carbon, nitrogen, and phosphorus in native and cultivated soils. Soil Sci. Soc. Am. J. 50, 627 - 633.

Eynard, A., Schumacher, T.E., Lindstrom, M.J., Malo, D.D., Kohl, R.A. 2006. Effects of aggregate structure and organic $\mathrm{C}$ on wettability of Ustolls. Soil Till. Res. 88, 205 - 216.

Garten Jr, C.T. 2011. Comparison of forest carbon dynamics at five sites along a latitudinal gradient. Geoderma. 167 -168, 30 - 40.

Gershenson, A., Bader, N.E., Cheng, W. 2009. Effects of substrate availability on the temperature sensitivity of soil organic matter decomposition. Global Change Biol. 15, 176 - 183.

Giardina, C.P., Ryan, M.G. 2000. Evidence that decomposition rates of organic carbon in mineral soil do not vary with temperature. Nature. 404, 858-861.

Han, K.H., Ha, S.G., Jang, B.C., 2010. Aggregate stability and soil carbon storage as affected by different land use practices. Proc. Int. Workshop on Evaluation and Sustainable Management of Soil Carbon Sequestration in Asian Countries. Bogor, Indonesia Sept. 28- 29.

Houghton, R.A. 2007. Balancing the global carbon budget. Annu. Rev. Earth Planet. Sci. 35, 313 - 347.
Hulscher, T.E.M., Cornelissen, G. 1996. Effect of temperature on sorption equilibrium and sorption kinetics of organic micropollutants: a review. Chemosphere. 32, 609 - 626.

Imriskova, I., Arreguín- Espinosa, R., Guzmán, S., Rodriguez-Sanoja, R., Langley, E., Sanchez, S. 2005. Biochemical characterization of the glucose kinase from Streptomyces coelicolor compared to Streptomyces peucetius var. caesius. Res. Microbiol. 156, 361- 366.

Kirschbaum, M.U.F. 1995. The temperature dependence of soil organic matter decomposition, and the effect of global warming on soil organic C storage. Soil Biol. Biochem. 27, 753- 760 .

Knorr, W., Prentice, I.C., House, J.I., Holland, E.A. 2005. Long-term sensitivity of soil carbon turnover to warming. Nature. 433, 298 - 301.

Kong, A.Y.Y., Six, J., Bryant, D.C., Denison, R.F., Kessel, C.V. 2005. The Relationship between Carbon Input, Aggregation, and Soil Organic Carbon stabilization in Sustainable Cropping Systems. Soil Sci. Soc. Am. J. 69, 1078 - 1085.

Lal, R., 1999. Soil compaction and tillage effects on soil physical properties of a Mallic Ochraqualf in northwest Ohio. J. Sustain. Agric. 14, 5365.

McLaren, R.G., Cameron, K.C. 1996. Soil Science. 2nd edition. Oxford University Press.

Mendes, I.C., Mandick, A.K., Dick, R.P., Bottomley, P.J. 1999. Microbial biomass and activities in soil aggregates affected by winter cover crops. Soil Sci. Soc. Am. J. 63, 873- 881.

Munkholm, L.J. 2011. Soil friability: A review of the concept, assessment and effects of soil properties and management. Geoderma. 167 $168,236-246$. 
Paustian, K., Cole, C.V., Sauerbeck, D.R., Sampson, N. 1998. $\mathrm{CO}_{2}$ mitigation by agriculture: an overview. Climatic Change. 40, 135- 162.

Reichstein, M., Kätterer, T., Andre, O., Ciais, P., Schulze, E.D., Cramer, W., Papale, D., Valentini, R. 2005. Does the temperature sensitivity of decomposition vary with soil organic matter quality?. Biogeosci. Discuss. 2, 737- 747.

Six J., Bossuyt, H., Degryze, S., Denef, K. 2004. A history of research on the link between (micro) aggregates, soil biota, and soil organic matter dynamics. Soil Till. Res. 79, 7 - 31.

Six, J., Feller, C., Denef, K., Ogle, S. M., De Moraes Sa, J.C., Albrecht, A. 2002. Soil organic matter, biota and aggregation in temperate and tropical soils: Effects of no-tillage. Agronomie. 22, 755-775.

Six, J., Elliott, E.T., Paustian, K. 2000. Soil macroaggregate turnover and micro-aggregate formation: A mechanism for $\mathrm{C}$ sequestration under no-tillage agriculture. Soil Biol. Biochem. 32, 2099- 2103.

Six, J., Elliott, E.T., Paustian, K., Doran, J.W. 1998. Aggregation and soil organic matter accumulation in cultivated and native grassland soils. Soil Sci. Soc. Am. J. 62, 1367 - 1377.
Swanston, C.W., Caldwell, B.A., Homann, P.S., Ganio, L., Sollins, P. 2002. Carbon dynamics during a long-term incubation of separate and recombined density fractions from seven forest soils. Soil Biol. Biochem. 34, 1121 - 1130.

Von Lützow, M., Kögel-Knabner, I. 2009. Temperature sensitivity of soil organic matter decomposition-what do we know?. Biol. Fertil. Soils. 46, 1-15.

Von Lützow, M., Kögel-Knabner, I., Ekschmitt, K., Matzner, E., Guggenberger, G., Marschner, B., Flessa, H. 2006. Stabilization of organic matter in temperate soils: Mechanisms and their relevance under different soil conditions: A review. Eur. J. Soil Sci. 57, 426- 445.

Walkley, A., Black, I.A. 1934. An examination of the Degtjareff method for determining soil organic matter and a proposed modification of the chromic acid titration method. Soil Sci. 37, 29- 38.

Zheng, Z.C., He, S.Q., Li, T.X., Wang Y.D., 2011. Effect of land use patterns on stability and distributions of organic carbon in the hilly region of Western Sichuan, China. Afr. J. Biotechnol. 10, 13107-13114. 